\title{
Influence of pCP1NetB ancillary genes on the virulence of Clostridium perfringens poultry necrotic enteritis strain $\mathrm{CP} 1$
}

\author{
Hongzhuan Zhou ${ }^{1,2}$, Dion Lepp ${ }^{2}$, Yanlong Pei ${ }^{3}$, Mei Liu², Xianhua Yin², Rongcai Ma' ${ }^{1}$, John F. Prescott ${ }^{3}$ \\ and Joshua Gong ${ }^{2^{*}}$
}

\begin{abstract}
Background: Necrotic enteritis (NE) is an economically important disease of poultry caused by certain Clostridium perfringens type A strains. The NetB toxin plays a critical role in the pathogenesis of NE. We previously demonstrated that netB is located within a $42 \mathrm{~kb}$ plasmid-encoded pathogenicity locus (NELoc-1), which also encodes 36 additional genes. Although NetB clearly plays a role in pathogenesis, the involvement of the other NELoc-1 genes has not yet been established. The current study was to provide experimental evidence to confirm the involvement of these genes in NE pathogenesis.
\end{abstract}

Results: The present study has characterized a virulent C. perfringens strain (CP1) that has spontaneously lost the NELoc-1-encoding plasmid, pCP1netB. When assessed for cytotoxicity on Leghorn Male Hepatoma (LMH) cells, the culture supernatant of the $\mathrm{PCP} 1$ netB-deficient $C P 1$ variant (CP1 $\triangle \mathrm{pCP} 1$ netB) demonstrated significantly reduced cytotoxicity compared to the wild-type. In addition, CP1 $\triangle \mathrm{pCP} 1$ netB was unable to cause intestinal lesions in chickens in a NE disease model. When netB alone was introduced into CP1 $\triangle \mathrm{PCP} 1$ netB, in vitro cytotoxicity was restored to the wild-type level; however, it did not completely restore virulence when used to challenge broiler chickens [mean lesion score of 0.71 compared to 3.23 in the wild type control group $(n=14)]$.

Conclusions: The results of this study suggest that other genes present in NELoc- 1 , in addition to netB, are required for full virulence in the chicken challenge model.

Keywords: Necrotic enteritis, Clostridium perfringens, Plasmid-deficient mutant, NELoc-1, NetB

\section{Background}

Avian necrotic enteritis (NE) is caused by certain strains of type A Clostridium perfringens, an anaerobic Gram-positive bacterium. It is of significant economic importance to the poultry industry, estimated to cost approximately US $\$ 6$ billion worldwide in 2015 [1]. The necrotic enteritis beta toxin-like (NetB) toxin was recently identified and shown to be a critical virulence factor in NE pathogenesis [2]. In these studies, a netB knockout mutant was unable to induce disease in an NE challenge chicken model system, while complementation

\footnotetext{
*Correspondence: joshua.gong@agr.gc.ca

${ }^{2}$ Guelph Research and Development Centre, Agriculture and Agri-Food Canada, 93 Stone Road West, Guelph, ON N1G 5C9, Canada

Full list of author information is available at the end of the article
}

with netB restored the virulence of the mutant [2]. Furthermore, several studies have examined $n e t B$ prevalence in a wide variety of $C$. perfringens strains and found a strong correlation between the presence of netB and host disease status [3-6]. Recently, we took the approach of comparative genomics and identified a genetic "signature" of NE disease in chickens [7]. We found that netB resides on a $42 \mathrm{~kb}$ pathogenicity locus, NELoc-1, located on a plasmid (pNetB) and associated specifically with virulent strains. This locus contains 36 genes in addition to $n e t B$, several of which are predicted to play a role in virulence. Two other loci (NELoc-2 and -3) associated with NE-producing strains were also identified: NELoc-2 consists of 11 genes and is chromosomally-encoded, whereas NELoc-3 consists of five genes and is located on 
a plasmid (pCpb2) distinct from that harboring NELoc1. The complete sequences of $\mathrm{pNetB}$ and $\mathrm{pCpb} 2$ from virulent strains originating in Canada and Australia have been reported and contain sequences with $>99 \%$ identity to NELoc-1 and NELoc-3, respectively $[8,9]$. The strict conservation of these loci suggests that they may encode genes important in the pathogenesis of NE. However, functional confirmation of these putative additional virulence factors has yet to be provided. The extrachromosomal location of most of the NE-associated genes (i.e. NELoc-1 and NELoc-3) presents an opportunity to remove these genes en masse, through plasmid loss, and thereby assess their collective role in virulence in a common genetic background. Furthermore, putative virulence genes may be reintroduced into the strains cured of their virulence plasmids, either individually or in combination, to assess each gene for phenotypic effects and possible interactions.

To extend our previous findings from the comparative genomic analyses, we have characterized a virulent C. perfringens strain (CP1) that has spontaneously lost its netB-carrying plasmid (pCP1netB). The virulence of the mutant, a netB-complemented derivative, and the wild-type strain has also been assessed both in vitro and in vivo. The results are reported herein.

\section{Methods}

\section{Bacterial strains, media and growth conditions}

Clostridium perfringens strain CP1 used in this study was a field isolate from an NE case in Ontario [10]. C. perfringens strains were routinely grown anaerobically at $37{ }^{\circ} \mathrm{C}$ in Brain Heart Infusion (BHI), Tryptone Proteose peptone Glucose (TPG), or Fluid Thioglycollate (FTG) broth (Difco). C. perfringens media were supplemented with $34 \mu \mathrm{g} / \mathrm{ml}$ chloramphenicol (Sigma-Aldrich, St. Louis, MO, USA) as required to retain the plasmid pJIR750::netB that carries a chloramphenicol resistance gene. E. coli Top 10 was used for cloning and grown at $37^{\circ} \mathrm{C}$ in Luria-Bertani (LB) broth or agar (Difco), supplemented with $34 \mu \mathrm{g} / \mathrm{ml}$ chloramphenicol as required.

\section{Pulsed field gel electrophoresis (PFGE)}

Pulsed field gel electrophoresis was performed on chromosomal and plasmid DNA as previously described [7]. DNA plugs for PFGE were prepared from overnight cultures of $C$. perfringens grown in BHI and the bacterial pellets incorporated into a final agarose concentration of $1 \%$ in PFGE-certified agarose (Bio-Rad, Hercules, CA, USA). Plugs were incubated overnight with gentle shaking at $37{ }^{\circ} \mathrm{C}$ in lysis buffer (0.5 M EDTA pH 8.0, 2.5\% of $20 \%$ sarkosyl), $0.25 \%$ lysozyme (Sigma-Aldrich) and subsequently incubated in $2 \%$ proteinase $\mathrm{K}$ (Roche, Laval, QC, Canada) buffer for 2 days at $55{ }^{\circ} \mathrm{C}$. Then plugs were equilibrated in $200 \mu \mathrm{l}$ restriction buffer at room temperature for $20 \mathrm{~min}$ and digested with $10 \mathrm{U}$ of NotI (New England Biolabs, Ipswich, MA) at $37{ }^{\circ} \mathrm{C}$ overnight. Electrophoresis was performed in a $1 \%$ PFGE-certified gel and separated with the CHEF-III PFGE system (Bio-Rad) in $0.5 \mathrm{X}$ Tris-borate-EDTA buffer at $14{ }^{\circ} \mathrm{C}$ at $6 \mathrm{~V}$ for $19 \mathrm{~h}$ with a ramped pulse time of 1-12 s. Gels were stained in ethidium bromide and visualized by UV light. Mid-Range II PFG markers (New England Biolabs) were used as a molecular DNA ladder.

\section{Genome sequencing and phylogenetic analysis}

Total genomic DNA was isolated from strains CP1 and $\mathrm{CP} 1 \triangle \mathrm{pCP} 1$ netB using the Gentra Puregene kit (Qiagen). Sequencing libraries were prepared with the Nextera XT DNA sample preparation kit (Illumina, San Diego, CA) according to the manufacturer's instructions, using 1 ng of total gDNA as input. The library concentration was determined by quantitative PCR, and diluted to a final concentration of $8 \rho \mathrm{p}$ in $10 \mathrm{mM}$ Tris $-\mathrm{HCl}$, $\mathrm{pH}$ 8. Sequencing was performed on a MiSeq instrument (Illumina) using the 600v3 kit, to generate $300 \mathrm{bp}$ paired-end reads. The quality of the resulting Fastq files was assessed with FastQC (http://www.bioinformatics. bbsrc.ac.uk/projects/fastqc), and reads were quality filtered and assembled with SPAdes v3.0 [11]. Phylogenetic trees based on whole genome sequence data were built using REALPHY [12], which uses Bowtie 2 [13] for mapping reads to a reference genome and RAxML [14] for tree-building. The ATCC13124 genome (gi_110798562) was used as a reference and C. perfringens strains ATCC3626 (gi_151558394), JGS1721 (gi_177911222), F262 (gi_422872590), NCTC8239 (gi_151558627), WAL-14572 (gi_373228597), JGS1987 (gi_151558496), JGS1495 (gi_151558295), Str.13 (gi_18308982), F4969 (gi_151558571), 1207_CPER (gi_875303967), JJC (gi_558869255), and CP4 (gi_942714078) were also included in the comparison.

\section{Construction of plasmid pJIR750::netB and complementation of $C P 1 \triangle \mathrm{pCP} 1$ netB}

The net $B$ complementation plasmid pJIR750::netB was constructed using primers CTC_netBF (CGGGATCCGTACCATTTAAATTAAGCAC) and CTC_netBR (TCGAGCTCCCCTCTATATACTATTGATTG), which contain BamHI and SacI restriction sites (underlined), respectively. They were used to amplify a $1551 \mathrm{bp}$ fragment encompassing the wildtype netB gene and $300 \mathrm{bp}$ of upstream sequence. Following digestion with BamHI and $S a c \mathrm{I}$, the fragment was purified with the Qiagen PCR purification kit (Qiagen, Mississauga, Ontario, Canada), and ligated into the $\mathrm{BamHI} / \mathrm{SacI}$ sites of the E. coli-C. perfringens shuttle vector pJIR750 (a gift from 
Dr. Julian I. Rood, Monash University, Australia), which confers chloramphenicol resistance, and the resulting pJIR750::netB plasmid was confirmed by sequencing. pJIR750::net $B$ was then introduced into $C P 1 \Delta \mathrm{pCP} 1$ netB by electroporation to generate $\mathrm{CP} 1 \Delta \mathrm{pCP} 1$ net $\mathrm{B}\left(\right.$ net $\left.B^{+}\right)$. The transformation mixture was plated onto $\mathrm{BHI}$ agar supplemented with $34 \mu \mathrm{g} / \mathrm{ml}$ chloramphenicol and incubated overnight at $37{ }^{\circ} \mathrm{C}$ under anaerobic conditions. The $\mathrm{CP} 1 \Delta \mathrm{pCP} 1$ netB $($ net $B+)$ transformants were confirmed by PCR amplification of a $302 \mathrm{bp}$ fragment using primers QnetBF (AGTGTAATTAGTACAAGCC) and QnetBR (GGCCATTTCATTTTTCCGTAA) and the following cycling conditions: initial denaturation at $94^{\circ} \mathrm{C}$ for $5 \mathrm{~min}$; 30 cycles at $94{ }^{\circ} \mathrm{C}$ for $30 \mathrm{~s}, 47^{\circ} \mathrm{C}$ for $30 \mathrm{~s}$ and $68{ }^{\circ} \mathrm{C}$ for $25 \mathrm{~s}$; final extension at $68^{\circ} \mathrm{C}$ for $10 \mathrm{~min}$.

\section{LMH cytotoxicity assay}

Lactate dehydrogenase (LDH) cytotoxicity assays were performed essentially as described [15, 16]. Briefly, chicken Leghorn male hepatoma (LMH) cells (ATCC CRL-2117) were maintained in Earl's minimum essential medium (EMEM) (Invitrogen) supplemented with L-glutamine, MEM Non-Essential Amino Acids Solution, 100 $\mathrm{U} / \mathrm{ml}$ penicillin, $100 \mu \mathrm{g} / \mathrm{ml}$ streptomycin and $10 \%$ fetal bovine serum. Flasks and 96-well plates were pre-coated with $0.1 \%$ gelatine (Millipore, Billerica, MA, USA) for adherence to surfaces. Cells were incubated in a humidified environment of $5 \% \mathrm{CO}_{2}$ at $37^{\circ} \mathrm{C}$.

For the cell cytotoxicity assays, 96-well cell culture plates (BD Biosciences) were seeded with $\sim 4 \times 10^{4} \mathrm{LMH}$ cells/well and incubated for $2-3$ days until just $100 \%$ confluent. C. perfringens strains were grown in TPG broth to an $\mathrm{OD}_{600 \mathrm{~nm}}$ of $0.6-0.8$, and broth culture supernatants were obtained by centrifugation at $18,000 \times g$ for $15 \mathrm{~min}$. The supernatants were filter-sterilized and added to the LMH cell medium (all samples were performed in triplicate), then incubated for $4 \mathrm{~h}$ at $37^{\circ} \mathrm{C}$ with $5 \% \mathrm{CO}_{2}$. Lactate dehydrogenase (LDH) release into the supernatant was measured as an indicator of cytolysis using the CytoTox Non-Radioactive (Promega) kit. Triton-X 100 (1\%) was used as positive control, which gave $100 \%$ cell death; the experiment was repeated three times.

\section{RT-PCR}

Total RNA was isolated from overnight cultures of C. perfringens strains using RiboPure ${ }^{\mathrm{TM}}$-Bacteria Kit (Applied Biosystems) and DNase treated with the Ambion Turbo DNase kit (BioRad). All first-strand cDNAs were prepared from same amount of approximately $2.5 \mu \mathrm{g}$ of total RNA in a $20 \mu \mathrm{l}$ reaction containing $0.5 \mathrm{mM}$ dNTPs, $1 \mu \mathrm{g}$ random hexamers (Invitrogen), $1 \times$ First strand buffer, $10 \mathrm{mM}$ dithiothreitol, $40 \mathrm{U}$ RNasin (Promega) and $200 \mathrm{U}$ Superscript II (Invitrogen). The reaction was incubated at
$25{ }^{\circ} \mathrm{C}$ for $10 \mathrm{~min}, 42{ }^{\circ} \mathrm{C}$ for $50 \mathrm{~min}$ and $70{ }^{\circ} \mathrm{C}$ for $15 \mathrm{~min}$. PCR amplification of transcribed netB was carried out using $2 \mu \mathrm{l}$ of cDNA as template and primers QnetBF and QnetBR as described above.

\section{Infection of chickens}

Fifty-four commercial 1-day-old male white Plymouth Rock broiler chickens (Bonnie's Chick Hatchery, Elmira, ON, Canada) were randomly divided into 4 experimental groups ( $\mathrm{n}=13-14$ per group). The chickens were fed an antibiotic-free chicken starter containing $20 \%$ protein for 11 days followed by turkey starter containing $28 \%$ protein (Arkell Research Station, University of Guelph). For the experimental infection (challenge) of birds, wild type $\mathrm{CP} 1, \mathrm{CP} 1 \Delta \mathrm{pCP} 1$ netB and $\mathrm{CP} 1 \Delta \mathrm{pCP} 1$ netB $\left(\right.$ net $\left.^{+}\right)$ were grown in cooked meat medium (Difco) (CMM) for $24 \mathrm{~h}$ at $37{ }^{\circ} \mathrm{C}$ under anaerobic conditions. FTG medium (Difco) was then inoculated $3 \%(\mathrm{v} / \mathrm{v})$ with an overnight CMM culture and incubated aerobically at $37^{\circ} \mathrm{C}$ for $15 \mathrm{~h}$. The growth at $15 \mathrm{~h}$ was approximately $\log _{10} 8.45 \pm 0.14$ C. perfringens colony-forming units (CFUs) per ml. The FTG-grown culture was then mixed with feed at a ratio of $2: 1(\mathrm{v} / \mathrm{w})$. The chickens were starved for $24 \mathrm{~h}$ before the $C$. perfringens contaminated feed was administered on day 13. Inoculated feed was prepared fresh twice daily and fed to chickens for 5 days.

Chickens were euthanized with carbon dioxide on the day following a 5-day challenge, and at necropsy the small intestine was examined for grossly visible lesions. Necrotic enteritis lesions were scored blindly using the system described by Keyburn et al. [2]

To determine if there were differences in the number of birds with lesions between two challenge groups, a two-tailed Fisher's exact test was used to assess the null hypothesis that the proportion of birds with lesions among the two groups was the same. The null hypothesis was rejected at $\mathrm{p} \leq 0.05$.

\section{Results \\ Identification of a CP1 isolate deficient in PCP1netB}

Type A strain CP1 was chosen as the potential transformable host for transformation experiments. Of particular interest, after inoculation of a CP1 stock culture from $-80{ }^{\circ} \mathrm{C}$ and several in vitro sub-cultures in $\mathrm{BHI}$ broth, we obtained one net $B$ negative isolate which was also negative for several other genes located on NELoc-1 locus. This strain was named $\mathrm{CP} 1 \Delta \mathrm{pCP} 1$ netB and its identification as a derivative of the virulent strain $\mathrm{CP} 1$ deficient in the plasmid (pCP1netB) that carries NELoc-1 was subsequently confirmed. Previously, our group reported that NELoc-1 is located on a $\sim 85 \mathrm{~kb}$ plasmid in CP1, the largest of at least four plasmids carried by this strain. PFGE analysis of plasmid DNA from 
$\mathrm{CP} 1$ and $\mathrm{CP} 1 \Delta \mathrm{pCP} 1$ netB confirmed that the $\mathrm{pCP} 1$ netB had been lost. As expected, the plasmid PFGE profile of $\mathrm{CP} 1 \triangle \mathrm{pCP} 1$ netB differed from the wildtype CP1 by the absence of the largest $85 \mathrm{~kb}$ plasmid band (Fig. 1a). This missing $85 \mathrm{~kb}$ band corresponded to the same size band previously shown, through Southern blotting experiments, to hybridize with a netB Dig-labelled probe. PFGE analysis of SmaI-digested chromosomal DNA from CP1 and $\mathrm{CP} 1 \triangle \mathrm{pCP} 1$ netB revealed that the two strains otherwise share an identical PFGE pattern, with the exception of the absent band in the mutant that corresponds in size to pCP1netB (Fig. 1b).

To further confirm that $\mathrm{CP} 1 \triangle \mathrm{pCP} 1$ netB was a derivative of CP1, genomic DNA from both isolates was sequenced using high throughput sequencing. Phylogenetic analysis of the two genomes, along with the genomes of 13 other $C$. perfringens strains currently available in GenBank, was performed using REALPHY, using ATCC 13124 as a reference genome. This tool reconstructs phylogeny based on single-nucleotide polymorphisms (SNPs) of conserved whole genome alignments, and is suitable for distinguishing closely related bacterial strains. As expected, $\mathrm{CP} 1$ and $\mathrm{CP} 1 \triangle \mathrm{pCP} 1$ netB were indistinguishable from each other using this method, and were closely related to $\mathrm{CP} 4$, another poultry strain isolated from the same time and location as CP1 (Fig. 2). Other C. perfringens strains, though still closely related, were clearly separated into different branches on the phylogenetic tree. Mapping of the $\mathrm{CP} 1 \Delta \mathrm{pCP} 1$ netB reads to

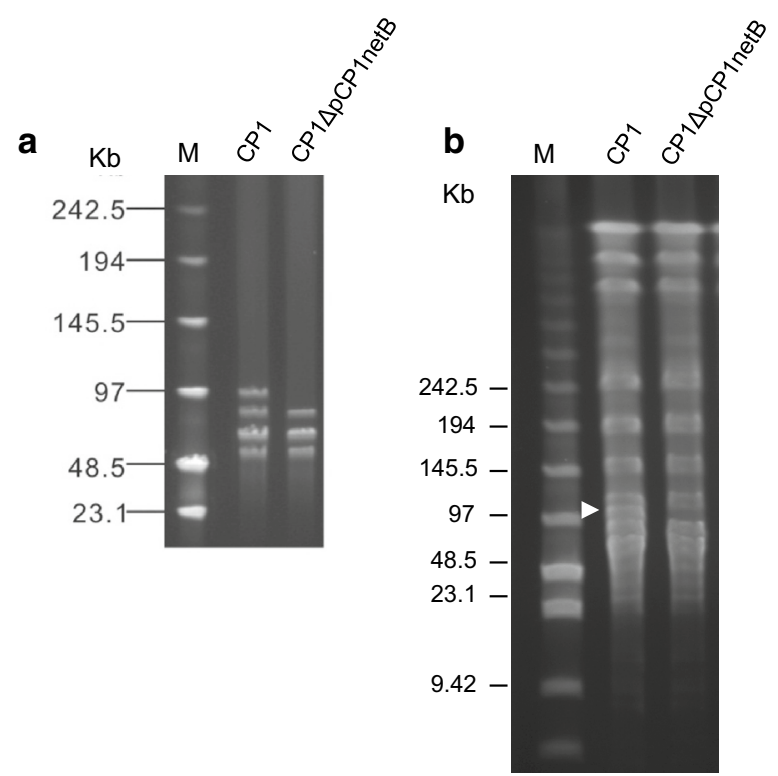

Fig. 1 a PFGE profiles of large plasmids from $C P 1$ and $C P 1 \triangle p C P 1$ netB (digested with Not I). $\mathbf{b}$ Chromosomal Smal-PFGE profiles of CP1 and $C P 1 \triangle P C P 1$ netB. The arrowhead indicates the band missing in CP1 $\triangle \mathrm{pCP} 1$ netB. $M$ mid-range II PFG markers the pNetB-NE10 sequence (JQ655731.1) also confirmed the absence of the NELoc1 pathogenicity locus (data not shown).

\section{Complementation of mutant $\mathrm{CP} 1 \Delta \mathrm{pCP} 1$ netB with netB alone}

In order to isolate the contribution of netB alone to the virulence of $\mathrm{CP} 1$, in the absence of other putative NELoc-1 virulence genes, a complemented strain $\mathrm{CP} 1 \Delta \mathrm{pCP} 1$ netB $\left(\right.$ net $\left.B^{+}\right)$was prepared by electroporating the mutant with pJIR750::netB, a pJIR750 derivative encoding the wildtype $C$. perfringens net $B$ gene. When RT-PCR was performed to evaluate netB expression in these strains, both wildtype CP1 and the complemented strain supported amplification of a $302 \mathrm{bp}$ netB product (Fig. 3). In contrast, no RT-PCR product was detected using template RNA isolated from the mutant. These results confirmed the expression of netB in the complemented strain.

\section{Cytotoxic effects towards LMH cells}

The NetB toxin has specific in vitro cytotoxic effects against the LMH cell-line. To quantitate the cytotoxicity of wildtype $\mathrm{CP} 1$, the mutant and the complemented strain, culture supernatants of these isolates were tested in the LMH cell cytotoxicity assay. The results showed that the level of cytotoxicity exhibited by culture supernatant derived from the mutant was significantly reduced compared to the wildtype control (Fig. 4). In contrast, complementation of the mutant with netB restored cytotoxicity levels to that found in CP1.

\section{Virulence of $\mathrm{CP} 1 \Delta \mathrm{pCP} 1$ netB and $\mathrm{CP} 1 \Delta \mathrm{pCP} 1$ netB net $^{+}$) in vivo}

To determine the potential of netB alone to cause NE, four groups of broiler chickens were challenged with the wildtype, the mutant, and the complement of CP1 or FTG media alone in an NE infection model. No lesions were observed in birds fed with FTG medium (Control) or the mutant (Fig. 5). By contrast, significant levels of disease were detected in birds infected with the wildtype $\mathrm{CP} 1$, with an average lesion score of 3.23. In the group infected with the complemented pNetB-negative isolate, five out of 14 birds had lesion score of 2 , generating an average lesion score of 0.71 . The proportion of infected birds in the complemented group was significantly less (Fisher's exact test, $\mathrm{p} \leq 0.05$ ) than that of the wildtype CP1 group.

\section{Discussion}

In this study, we have characterized a $C$. perfringens mutant from a chicken NE strain (CP1) that spontaneously lost the plasmid carrying the NE-specific locus 


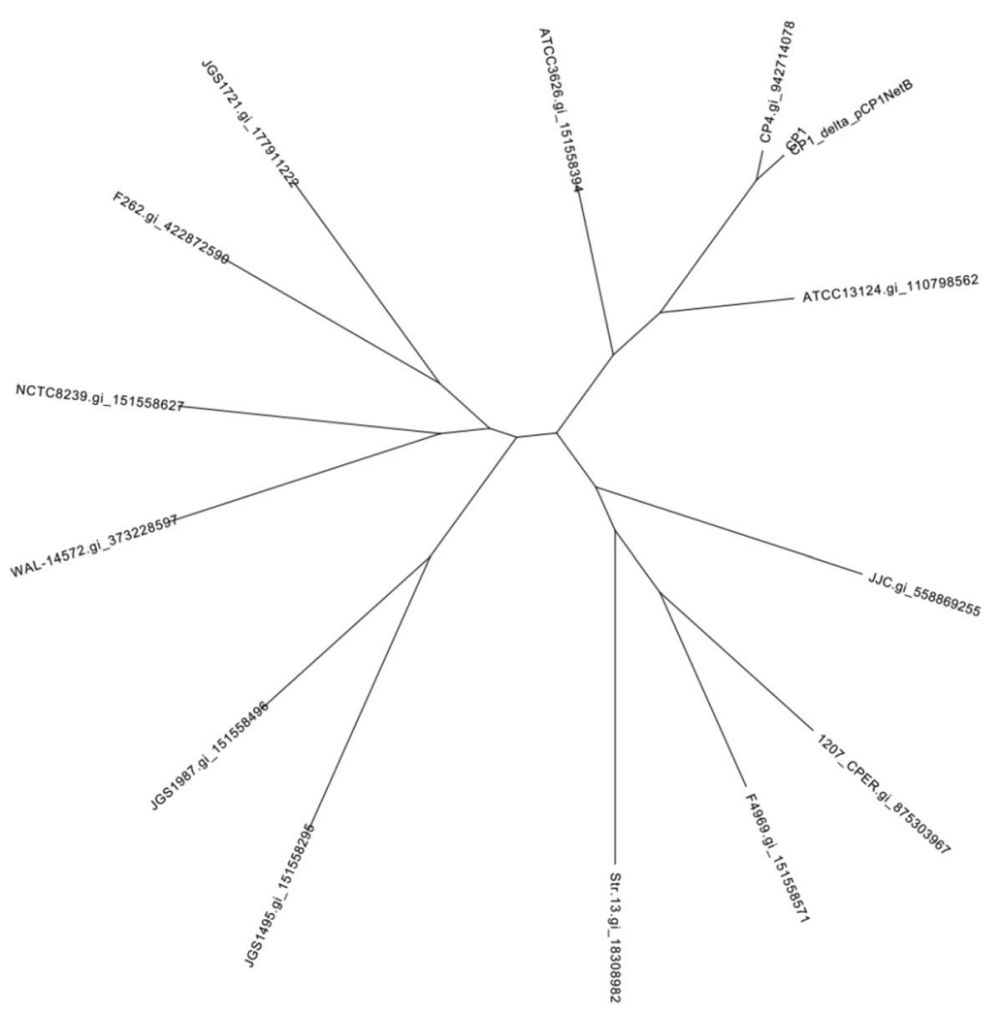

Fig. 2 Phylogenetic tree based on whole genome alignments of CP1, CP1 $\triangle \mathrm{pCP} 1$ netB generated with REALPHY. Whole genome sequences of CP1, $\mathrm{CP} 1 \triangle \mathrm{PCP} 1$ netB and 12 additional C. perfringens genomes currently available in Genbank were aligned with REALPHY and a phylogenetic tree generated with RAxML. Strain names and gene accession numbers are given for each genome

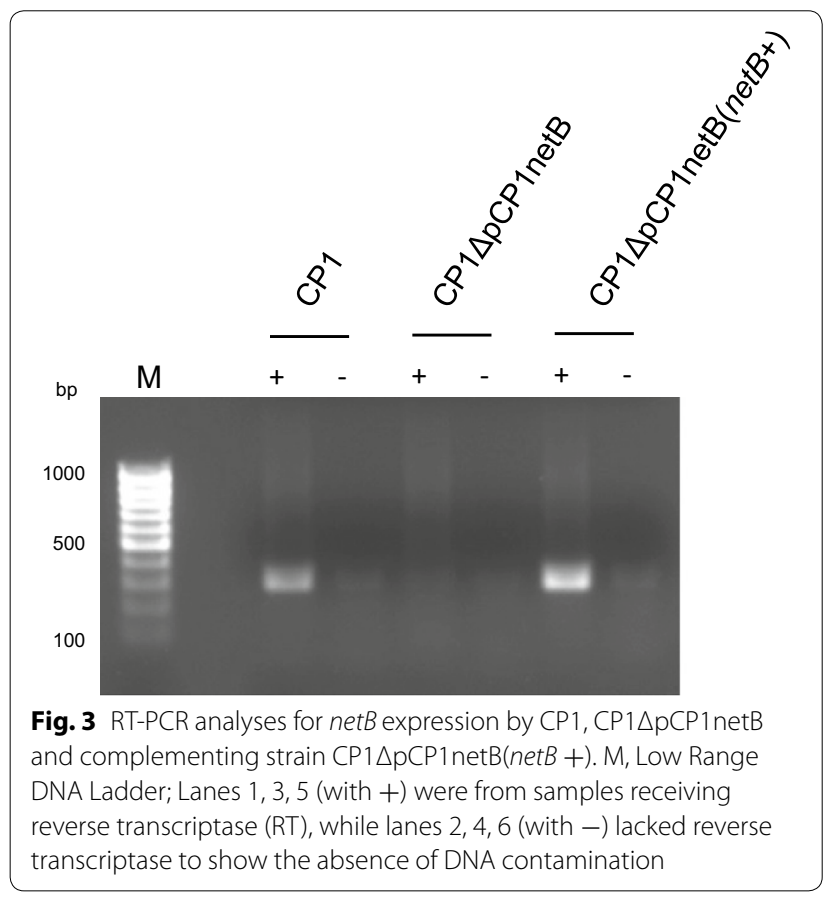

NELoc-1, including investigating its cytotoxicity towards the chicken male Leghorn hepatoma cell-line and capacity to produce NE lesions in broilers. The validity of this $\mathrm{CP} 1$ mutant $(\mathrm{CP} 1 \triangle \mathrm{pCP} 1$ netB) as lacking only the $\mathrm{pNetB}$ plasmid and having no other gene changes was confirmed by PFGE of plasmid and chromosomal DNA and genome sequencing (Figs. 1, 2).

Previously, our group reported on three highly conserved NE-associated loci that were designated NELoc-1 (42 kb), NELoc-2 (11.2 kb) and NELoc-3 (5.6 kb). The largest locus, NELoc-1, consists of netB and 36 additional genes [7]. In the present study, we tested the virulence conferred by net $B$ alone, in the absence of the other NELoc-1 genes. The expression of netB was sufficient to restore the in vitro cytotoxicity of $\mathrm{CP} 1 \Delta \mathrm{pCP} 1$ netB to wildtype levels, as observed when supernatant from the complemented $\mathrm{CP} 1 \Delta \mathrm{pCP} 1$ netB $\left(\right.$ net $\left.B^{+}\right)$was tested in the LMH cytotoxicity assay (Fig. 4). Cytotoxicity of $C$. perfringens culture supernatants towards the LMH cellline has been demonstrated to be specifically attributable to NetB [17], and has been used extensively to assay the activity of NetB $[2,15,18]$. Our study importantly 


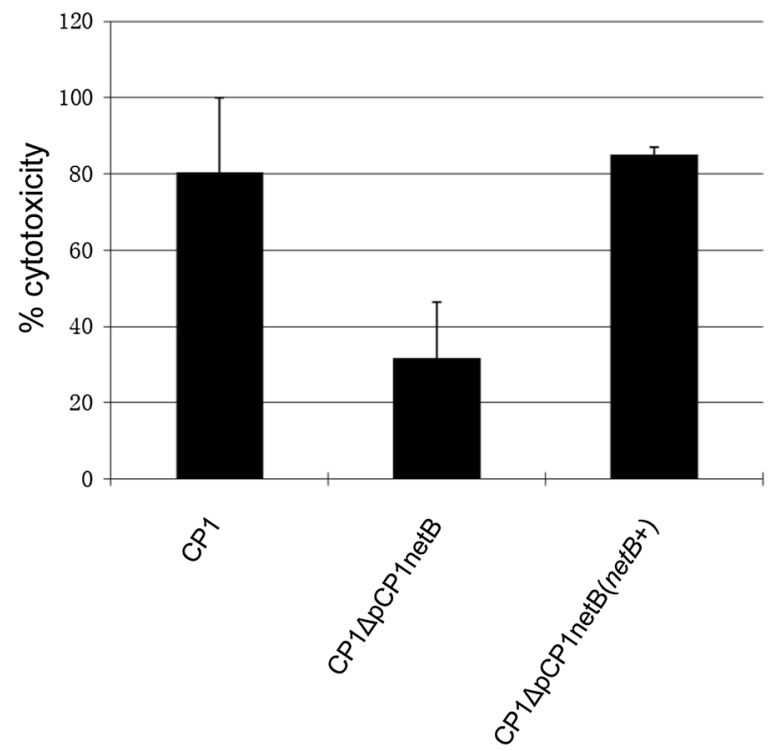

Fig. $4 \mathrm{LMH}$ cell cytotoxicity assay. Culture supernatants were isolated from $C P 1, C P 1 \triangle p C P 1$ netB and complementing strain $C P 1 \triangle \mathrm{PCP} 1$ netB(netB +$)$. The experiment was repeated three times, and average results are shown. Error bars depict standard deviation (SD). The amount of cytotoxicity induced by NetB in each cell supernatant is expressed as a percentage

suggests that genes on the pNetB pathogenicity island (other than $n e t B$ ) are not involved directly in regulating expression of NetB.

When assessed in vivo, the complemented group failed to fully restore the ability of $\mathrm{CP} 1 \triangle \mathrm{pCP} 1$ netB to cause NE (Fig. 5). While it has been reported that there is not always a direct correlation between in vitro cytotoxicity and the development of NE [19], the inability of net $B$ complementation alone to restore full virulence to $\mathrm{CP} 1 \triangle \mathrm{pCP} 1$ netB also suggests that other genes present on $\mathrm{pCP} 1$ netB are required during NE pathogenesis in a role not related to NetB expression. NELoc-1 encodes a number of genes with putative virulence-related functions, including adhesins, glycosidases, and a cyclic-diGMP signaling system [7], which plausibly influence the survival and colonization of $C$. perfringens in the intestinal mucosa during NE pathogenesis, as well as other possible functions. The fact that neither netB transcription determined through semi-quantitative RT-PCR, nor in vitro LMH cytotoxicity appeared to be reduced by $\mathrm{CP} 1 \Delta \mathrm{pCP} 1$ netB $\left(\right.$ net $\left.B^{+}\right)$, indicates that this isolate has the capacity similar to the wild-type strain in the production of NetB toxin. In addition, the uneven distribution of NE disease (approximately one-third of the birds showed NE disease but two-thirds had no NE lesion) and less severe $\mathrm{NE}$ lesions in the complemented group make it difficult to attribute the results to the loss of the vector, given

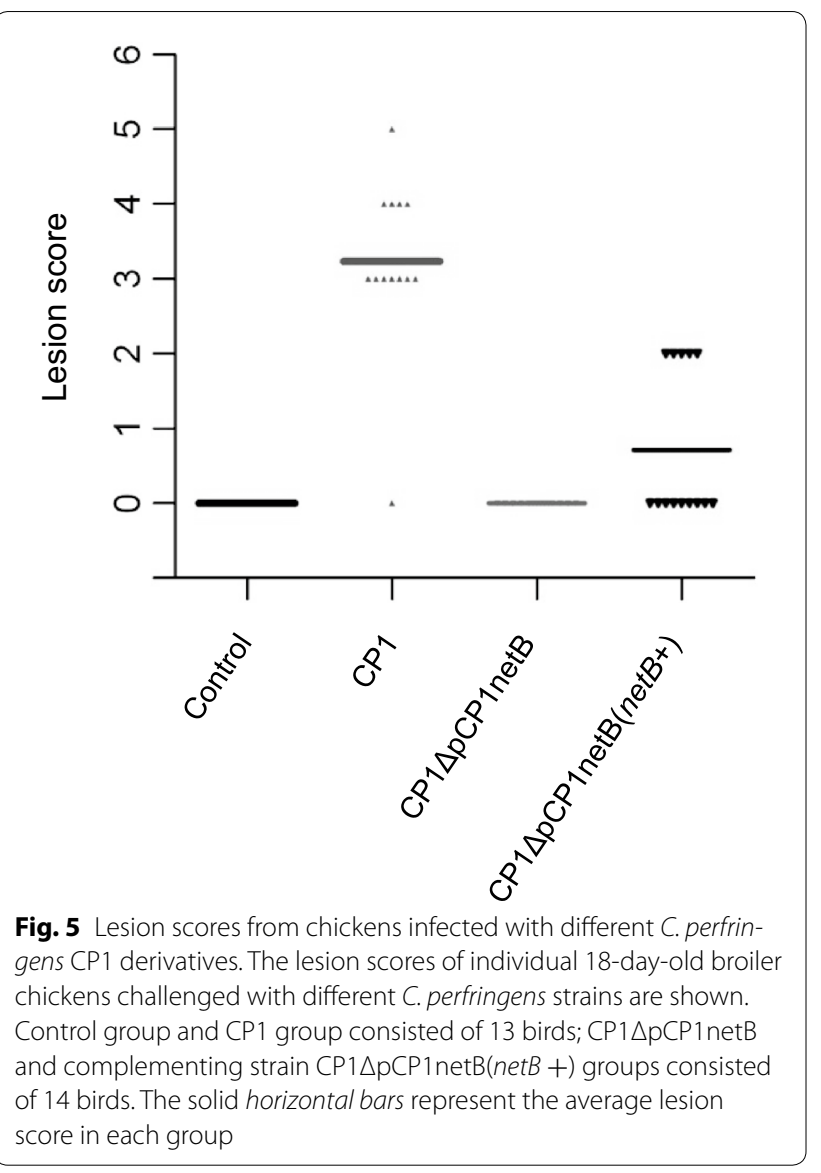

that all the birds received the same delivery of the isolate through a diet during the challenge. Thus, our results support the hypothesis that although NetB is the essential factor which initiates NE disease, its delivery to target cells clearly involves a complex delivery system likely including mucus and mucosal adhesion [21].

It should be noted that the disease model system employed in this study, and by others, may be insufficient for assessing the contribution to pathogenesis of all accessory virulence factors, which could each play a role at any point during the infection cycle of the pathogen $[20,21]$. For example, genes involved in environmental survival, transmission, competition with the indigenous intestinal microbiota, or other activities that may normally be essential for pathogenesis, may no longer be required due to the severe challenge used in this model system. Hence, more sensitive in vivo assays directly suited to evaluate these functions need to be explored.

\section{Conclusions}

The current study found that netB, in the absence of other NELoc-1 genes, was able to completely restore in vitro cytotoxicity, but only partially restore virulence 
in vivo. These results importantly support the hypothesis that other genes present on NELoc-1 are involved in NE pathogenesis and have functions other than controlling the expression of NetB.

\section{Authors' contributions}

$H Z, D L$, and JG designed the study and wrote the manuscript. $H Z$ and DL performed laboratory experiments and data analyses. JP and YL designed and conducted the chicken trial with support from other authors. ML identified CP1 strain as a transformable recipient of plasmids. XY and RM contributed to data analyses and discussion. JG supervised the research. All authors read and approved the final manuscript.

\section{Author details}

${ }^{1}$ Beijing Agro-Biotechnology Research Center, Beijing Academy of Agriculture and Forestry Sciences, Beijing 100097, China. ${ }^{2}$ Guelph Research and Development Centre, Agriculture and Agri-Food Canada, 93 Stone Road West, Guelph, ON N1G 5C9, Canada. ${ }^{3}$ Department of Pathobiology, University of Guelph, Guelph, ON N1G 2W1, Canada.

\section{Acknowledgements}

Plasmid pJIR750 was a gift from Dr. Julian I. Rood, Monash University, Australia. $\mathrm{HZ}$ was a visiting Ph.D. student to Guelph Research and Development Centre supported by the China Scholarship Council through the MOE-AAFC Ph.D. Student Research Program. ML was a NSERC Visiting Fellow to Canadian Federal Government Laboratories.

\section{Competing interests}

The authors declare that they have no competing interests.

\section{Availability of data and materials}

The data supporting the findings of this study are contained within the manuscript.

\section{Ethics approval and consent to participate}

The experiment with chickens and conditions for their use was approved by the University of Guelph Animal Care Committee (Reference\# AUP 1936) in accordance with the Canadian Council on Animal Care's Guidelines.

\section{Funding}

This work was supported by Agriculture and Agri-Food Canada and Canadian Poultry Research Council through the Poultry Cluster program. The funders had no role in study design, data collection and analysis, decision to publish, or preparation of the manuscript.

Received: 1 October 2016 Accepted: 15 December 2016

Published online: 21 January 2017

\section{References}

1. Wade B, Keyburn AL. The true cost of necrotic enteritis. World Poult. 2015;9:2.

2. Keyburn AL, Boyce JD, Vaz P, Bannam TL, Ford ME, Parker D, Di Rubbo A, Rood Jl, Moore RJ. NetB, a new toxin that is associated with avian necrotic enteritis caused by Clostridium perfringens. PLoS Pathog. 2008;4:e26.

3. Chalmers G, Bruce HL, Hunter DB, Parreira VR, Kulkarni RR, Jiang Y-F, Prescott JF, Boerlin P. Multilocus sequence typing analysis of Clostridium perfringens isolates from necrotic enteritis outbreaks in broiler chicken populations. J Clin Microbiol. 2008:46:3957-64.
4. Johansson A, Aspán A, Kaldhusdal M, Engström BE. Genetic diversity and prevalence of netB in Clostridium perfringens isolated from a broiler flock affected by mild necrotic enteritis. Vet Microbiol. 2010;144:87-92.

5. Keyburn AL, Yan XX, Bannam TL, Van Immerseel F, Rood Jl, Moore RJ. Association between avian necrotic enteritis and Clostridium perfringens strains expressing NetB toxin. Vet Res. 2010;41(2):1-8.

6. Keyburn AL, Bannam TL, Moore RJ, Rood JI. NetB, a pore-forming toxin from necrotic enteritis strains of Clostridium perfringens. Toxins. 2010;2:1913-27.

7. Lepp D, Roxas B, Parreira VR, Marri PR, Rosey EL, Gong J, Songer JG, Vedantam G, Prescott JF. Identification of novel pathogenicity loci in Clostridium perfringens strains that cause avian necrotic enteritis. PLoS ONE. 2010;5:e10795.

8. Bannam TL, Yan XX, Harrison PF, Seemann T, Keyburn AL, Stubenrauch C, Weeramantri LH, Cheung JK, McClane BA, Boyce JD, et al. Necrotic enteritis-derived Clostridium perfringens strain with three closely related independently conjugative toxin and antibiotic resistance plasmids. MBio. 2011;2:e00190-211.

9. Parreira VR, Costa M, Eikmeyer F, Blom J, Prescott JF. Sequence of two plasmids from Clostridium perfringens Chicken necrotic enteritis isolates and comparison with C. perfringens conjugative plasmids. PLoS ONE. 2012;7:e49753.

10. Thompson DR, Parreira VR, Kulkarni RR, Prescott JF. Live attenuated vaccine-based control of necrotic enteritis of broiler chickens. Vet Microbiol. 2006;113:25-34.

11. Bankevich A, Nurk S, Antipov D, Gurevich AA, Dvorkin M, Kulikov AS, Lesin VM, Nikolenko SI, Pham S, Prjibelski AD, et al. SPAdes: a new genome assembly algorithm and its applications to single-cell sequencing. J Comput Biol. 2012;19:455-77.

12. Bertels F, Silander OK, Pachkov M, Rainey PB, van Nimwegen E. Automated reconstruction of whole-genome phylogenies from shortsequence reads. Mol Biol Evol. 2014;31:1077-88.

13. Langmead B, Salzberg SL. Fast gapped-read alignment with Bowtie 2. Nat Methods. 2012;9:357-9.

14. Stamatakis A. RAxML-VI-HPC: maximum likelihood-based phylogenetic analyses with thousands of taxa and mixed models. Bioinformatics. 2006:22:2688-90.

15. Cheung JK, Keyburn AL, Carter GP, Lanckriet AL, Van Immerseel F, Moore RJ, Rood J. The VirSR two-component signal transduction system regulates NetB toxin production in Clostridium perfringens. Infect Immun. 2010;78:3064-72.

16. Zhu J, Yin X, Yu H, Zhao L, Sabour P, Gong J. Involvement of quorum sensing and heat-stable enterotoxin a in cell damage caused by a porcine enterotoxigenic Escherichia coli strain. Infect Immun. 2011;79:1688-95.

17. Keyburn AL, Yan XX, Bannam TL, Van Immerseel F, Rood Jl, Moore RJ. Association between avian necrotic enteritis and Clostridium perfringens strains expressing NetB toxin. Vet Res. 2010;41:21.

18. Lanckriet A, Timbermont L, Eeckhaut V, Haesebrouck F, Ducatelle R, Van Immerseel F. Variable protection after vaccination of broiler chickens against necrotic enteritis using supernatants of different Clostridium perfringens strains. Vaccine. 2010;28:5920-3.

19. Smyth JA, Martin TG. Disease producing capability of netB positive isolates of Clostridium perfringens recovered from normal chickens and a cow, and netB positive and negative isolates from chickens with necrotic enteritis. Vet Microbiol. 2010;146:76-84.

20. Prescott JF, Parreira VR, Mehdizadeh Gohari I, Lepp D, Gong J. The pathogenesis of necrotic enteritis in chickens: what we know and what we need to know: review. Avian Pathol. 2016;45:1-21.

21. Shojadoost B, Vince AR, Prescott JF. The successful experimental induction of necrotic enteritis in chickens by Clostridium perfringens: a critical review. Vet Res. 2012;43:74. 